\title{
Wavefront reconstruction in phase-shifting interferometry via sparse coding
}

\section{of amplitude and absolute phase}

\author{
V. Katkovnik ${ }^{1}$ and J. Bioucas-Dias ${ }^{2}$ \\ ${ }^{1}$ Department of Signal Processing, Technology University of Tampere, \\ Finland. E-mail: vladimir.katkovnik@tut.fi \\ ${ }^{2}$ Instituto de Telecomunicações and Instituto Superior Técnico, Universidade de Lisboa, Lisboa, \\ Portugal. E-mail: bioucas@lx.it.pt
}


Phase-shifting interferometry is a coherent optical method that combines high accuracy with high measurement speeds. This technique is therefore desirable in many applications such as efficient industrial quality inspection process. However, despite its advantageous properties, the inference of the object amplitude and the phase, herein termed wavefront reconstruction is not a trivial task owing to, namely, the Poissonian noise associated with the measurement process and to the $2 \pi$ phase periodicity of the observation mechanism. In this paper, we formulate the wavefront reconstruction as an inverse problem where the amplitude and the absolute phase are assumed to admit sparse linear representations in suitable sparsifying transforms (dictionaries). Sparse modeling is a form of regularization of inverse problems which, in the case of the absolute phase, is not available to the conventional wavefront reconstruction techniques as only interferometric phase modulo- $2 \pi$ is considered thereof. The developed sparse modeling of the absolute phase solves two different problems: essentially improved accuracy of the interferometric (wrapped) phase reconstruction and simultaneous phase unwrapping.

Based on this rationale, we introduce the sparse phase and amplitude reconstruction (SPAR) algorithm. SPAR takes into full consideration the Poissonian (photon counting) measurements and uses data adaptive BM3D frames as a sparse representation for the amplitude and for the absolute phase. SPAR effectiveness is documented by comparing its performance with that of competitors in a series of experiments. (c) 2014 Optical Society of America 


\section{Introduction}

The determination of the complex amplitude of a monochromatic light wave field, often termed wavefront reconstruction, is a fundamental step in many applications of optical metrology. Especially the phase distribution defined, e.g., in a plane, may be evaluated to yield information of the optical path the light has traveled before impinging on the sensor, e.g., a CCD camera. This knowledge enables a huge number of practical applications in industry and science, such as shape and deformation measurements down to a fraction of a micron or absolute distance measurements on an environmental scale [1].

There are various interferometric based methods aimed at determination of the complex amplitude of a monochromatic light wavefront, namely phase shifting, digital holography, and shearography [1], [2]. This paper is addressed to reconstruction of the object phase and amplitude in phase-shifting interferometry. In this technique, from each pixel of the object under study, we are given a set of noisy and nonlinear measurements of the complex field. The noise is Poissonian, linked with the photon counting process, and the nonlinearities are sinusoidal, each one corresponding to a different phase shift (more on the used phase-shifting interferometry in Section 3).

The sparse approximations of the wavefront proposed in this paper are quite general and can be used for various complex-valued wavefront reconstruction, thus the considered phaseshifting setting can be treated as a convenient scenario for clear demonstration of specific features of this technique. This paper is elaboration of the preliminary results introduced in [3] with respect to which we detail the adapted sparse model and the proposed multiobjective based optimization. In addition, we present a set of evidences providing clear effectiveness 
of the proposed methodology.

In what follows the paper is organized as follows. We start from the concept of the sparsity for the complex valued wavefront (Section 2), where it is emphasized the used sparsification of the phase as the absolute phase. The observation modeling for phase-shifting interferometry with the Poissonian noise are discussed in Section 3. The variational approach to the problem based on multiobjective optimization is considered in Section 4. The solutions of the considered variational problems are presented in the corresponding subsections 4.A, 4.B and 4.C. The developed iterative algorithm is discussed in Section 5. Experimental results are shown in Section 6 separately for visual evaluation of the phase and amplitude reconstruction (subsection 6.A) and for the numerical comparison of various techniques (subsection 6.C).

\section{Sparsity and low dimensional wavefront modeling}

In this paper, we use sparse modeling for wavefront reconstruction from phase-shifting interferometry measurements. It is well known that many images (and signals) admit sparse representations in the sense that they are well approximated by linear combinations of a small number of functions taken from a know set. On many occasions, this is a consequence of the self-similarity of these images: it is very likely to find in them many similar patches in different locations and at different scales. The topic of sparse and redundant representations has attracted tremendous interest from the research community in the last ten years. This interest stems from the role that the low dimensional models play in many signal and image areas such as compression, restoration, classification, and design of priors and regularizers, just to name a few [4].

Let $\mathbf{c} \in \mathbb{R}^{n}$ denote a vector representing an image, or a patch of it, and let us assume that c admits a sparse representation, or sparse coding, with respect to the columns of a given 
matrix $\boldsymbol{\Psi} \in \mathbb{R}^{n \times m}$; i.e., it is possible to write $\mathbf{c}=\boldsymbol{\Psi} \boldsymbol{\theta}$, where $\boldsymbol{\theta} \in \mathbb{R}^{m}$ is a vector containing only a few non-zero components. The matrix $\boldsymbol{\Psi}$ is termed a synthesis operator (or dictionary) because in the writing $\mathbf{c}=\sum_{j=1}^{m} \boldsymbol{\Psi}_{j} \theta_{j}$, where $\boldsymbol{\Psi}_{j}$ are the columns of the matrix $\boldsymbol{\Psi}$ and $\theta_{j}$ are the elements of the vector $\boldsymbol{\theta}, \mathbf{c}$ is synthesized as a linear combination of the columns of $\boldsymbol{\Psi}$ weighted by the elements of $\boldsymbol{\theta}$, often called the spectrum of $\mathbf{c}$. The synthesis based representations have a dual point of view in which, given an image $\mathbf{c} \in \mathbb{R}^{n}$, we compute its spectrum $\boldsymbol{\beta} \in \mathbb{R}^{m}$ by applying the so-called analysis operator (or dictionary) $\boldsymbol{\Phi} \in \mathbb{R}^{m \times n}$ to c, i.e. $\boldsymbol{\beta}=\boldsymbol{\Phi} \mathbf{c}$.

There are many classical transforms in which the direct transform $\boldsymbol{\Phi}$ is interpretable as an analysis operator and the inverse transform $\Psi$ as the corresponding synthesis operator. Quite often these matrices $\boldsymbol{\Phi}$ and $\boldsymbol{\Psi}$ are orthonormal, i.e., $\boldsymbol{\Phi}^{T} \boldsymbol{\Phi}=\mathbf{I}_{n \times n}$, where $\mathbf{I}_{n \times n}$ is the $n \times n$ identity matrix, and $\boldsymbol{\Psi}=\boldsymbol{\Phi}^{T}$. It happens, however that when we are looking for the sparsest approximation using a synthesis dictionary, the likelihood of success increases with the number and variety of signals (atoms) held by the dictionary. As a consequence, the synthesis dictionaries yielding sparse representations are often overcomplete, i.e., $m>n$. The concept of frame is a generalization of the classical basis especially developed for overcomplete (synthesis and analysis) representations with linearly dependent approximating functions [4].

Let $\mathbf{o} \in \mathbf{C}^{n}$ be a complex-valued wavefront defined on a grid with $n$ pixels. Denote $\mathbf{B}_{o}=$ $\bmod (\mathbf{o})$ and $\varphi_{o}=\operatorname{angle}(\mathbf{o}) \in[-\pi, \pi)$ and as, respectively, the corresponding images of amplitude (modulus) and the wrapped phase, $\boldsymbol{\varphi}_{o}$. Then we have $\mathbf{o}=\mathbf{B}_{o} \exp \left(j \boldsymbol{\varphi}_{o}\right)$. Herein, all functions applied to vectors are to be understood in the component-wise sense; the same applies to multiplications and divisions of vectors. In addition, if $\mathbf{o}$ is a vector and $a$ is a scalar, the notation $\mathbf{o} a$ stands for a vector with the same size of $\mathbf{o}$ such that its $i$-th component 
is given by $[\mathbf{o} a]_{i}=[\mathbf{o}]_{i} a$.

With the objective of formulating treatable phase imaging inverse problems, most approaches follow a two-step procedure: in the first step, an estimate of the so-called principal (wrapped, interferometric) phase in the interval $[-\pi, \pi)$ is determined; in the second step, termed phase unwrapping, the absolute phase is inferred by adding of an integer number of $2 \pi$ multiples to the estimated interferometric phase [5]. In what follows, we denote the principal phase as $\boldsymbol{\varphi}_{o}$ and the absolute phase as $\boldsymbol{\varphi}_{o, a b s}$. We introduce the phase-wrap operator $\mathcal{W}: \mathbb{R} \mapsto[-\pi, \pi)$, linking the absolute and principal phase as $\boldsymbol{\varphi}_{o}=\mathcal{W}\left(\boldsymbol{\varphi}_{o, a b s}\right)$. We also define the unwrapped phase as $\varphi_{o, a b s}=\mathcal{W}^{-1}\left(\varphi_{o}\right)$. Notice that $\mathcal{W}^{-1}$ is not the inverse function of $\mathcal{W}$ because the latter is not one-to-one and thus is does not have inverse.

In sparse coding for complex valued images, we may think in two different approaches: either we use a complex valued sparse representation to model directly the complex image $\exp \left(j \varphi_{o}\right)$, as recently proposed in [6], or we use sparse real valued representations for the amplitude $\mathbf{B}_{o}$ and absolute phase $\boldsymbol{\varphi}_{o, a b s}$ images of $\mathbf{o}$. The choice of the type of the sparse modeling depends on the application. The former is suited to wavefront reconstruction, where the interferometric (wrapped) phase carries all necessary phase information, whereas the latter is suited to applications requiring the inference of the absolute phase, herein termed phase reconstruction.

In this paper, we follow the second type of the wavefront modeling. We introduce formally this sparse wavefront modeling as follows:

$$
\begin{aligned}
& \mathbf{B}_{o}=\boldsymbol{\Psi}_{a, o} \boldsymbol{\theta}_{a, o}, \quad \boldsymbol{\varphi}_{o, a b s}=\boldsymbol{\Psi}_{\varphi, o} \boldsymbol{\theta}_{\varphi, o} \\
& \boldsymbol{\theta}_{a, o}=\boldsymbol{\Phi}_{a, o} \mathbf{B}_{0}, \quad \boldsymbol{\theta}_{\varphi, o}=\boldsymbol{\Phi}_{\varphi, o} \boldsymbol{\varphi}_{o, a b s}
\end{aligned}
$$

where $\boldsymbol{\theta}_{a, o}$ and $\boldsymbol{\theta}_{\varphi, o}$ are, respectively, the amplitude and phase (absolute phase) spectra of 
the object $\mathbf{o}$. In Eqs.(1), the amplitude $\mathbf{B}_{o}$ and absolute phase $\boldsymbol{\varphi}_{o, a b s}$ are synthesized from the amplitude and phase spectra $\boldsymbol{\theta}_{a, o}$ and $\boldsymbol{\theta}_{\varphi, o}$. On the other hand, the analysis Eqs.(2) give the spectra for amplitude and phase for the wavefront $\mathbf{o .}$

Following the rationale we have introduced in beginning of this section, we herein assume that the amplitude and phase (absolute phase) spectra, $\boldsymbol{\theta}_{a, o}$ and $\boldsymbol{\theta}_{\varphi, o}$, respectively, are sparse; i.e., most elements thereof are zero. In order to quantify the level of sparsity of $\boldsymbol{\theta}_{a, o}$ and $\boldsymbol{\theta}_{\varphi, o}$, i.e., their number of non-zero elements, we use the pseudo $l_{0}$-norm $\|\cdot\|_{0}$ defined as $\|\mathbf{x}\|_{0}=\left|\left\{i: x_{i} \neq 0, i=1, \ldots, n\right\}\right|$, where $\mathbf{x}$ is an $n$-dimensional vector. Therefore, in the ensuing formulations, we will design estimation criteria promoting low values of $\left\|\boldsymbol{\theta}_{a, o}\right\|_{0}$ and $\left\|\boldsymbol{\theta}_{\varphi, o}\right\|_{0}$. It is obvious that for the complex exponent there is no difference between the principal and absolute phase, $\exp \left(j \varphi_{o, a b s}\right)=\varphi\left(j \varphi_{o}\right)$, and the angle operator in $\varphi_{o}=\operatorname{angle}(\mathbf{o})$ gives the principal phase. However, there is a great deal of difference between the sparsity for the absolute and interferometric phases, because in many cases the absolute phase can be smooth or piece-wise smooth function easily allowing sparsification while the complex exponents is a periodic function of this phase.

The sparse approximation of the phase in the form Eq.(2) is initiated from our works [7]- [9], where it was presented for the interferometric phase assuming at least in the modeling that $-\pi \leq \varphi_{o, a b s}<\pi$. Formulation and detailed of this technique to the sparse approximation of the absolute phase which can take values out of the basic interval $[-\pi, \pi)$ is an essential step forward in this paper.

The approach proposed in this paper takes into full consideration the Poissonian (photon counting) measurements. In this way we are targeting at optimal sparse reconstruction both phase and amplitude taking into consideration all details of the observation process. 


\section{Phase-shifting interferometry}

Fig. 1 schematizes the phase-shifting interferometry concept. The light emitted by a laser is split into two beams by a beam splitter: one beam is directed towards the surface of an object under study; the other beam is directed towards a mirror which introduces a controlable phase shift. The intensity of the sum of these two beams is then measured by a sensor array. For the $L$-step phase-shifting interferometry, the complex-valued wavefront at the sensor plane is given by

$$
\mathbf{u}_{s}=\mathbf{B}_{o} \exp \left(j \varphi_{o}\right)+\mathbf{A}_{r} \exp \left(-j \varphi_{r_{s}}\right), s=1, \ldots, L
$$

where $\mathbf{B}_{o} \exp \left(j \boldsymbol{\varphi}_{o}\right)$ and $\mathbf{A}_{r} \exp \left(-j \varphi_{r_{s}}\right)$ are the object and reference wavefronts, respectively. The phase $\varphi_{r_{s}}$ is defined by the phase shift introduced by the mirror for $s=1, \ldots, L$.

Let us assume that the sensor takes measurements on a rectangular grid with $N$ digital elements and let $\mathbf{Y}_{s}=\left\{\mathbf{Y}_{s}[l], l=1, \ldots, N\right\}$, for $s=1, \ldots, L$, denote the $L$ measured images with $N$ elements each. The measurement process in optics amounts to count the photons hitting the sensor's elements and is well modeled by independent Poisson random variables; that is, the probability that $\mathbf{Y}_{s}[l]=k$, for a given non-negative integer $k$, is given by

$$
p\left(\mathbf{Y}_{s}[l]=k\right)=\exp \left(-\mathbf{I}_{s}[l] \chi\right) \frac{\left(\mathbf{I}_{s}[l] \chi\right)^{k}}{k !}
$$

where $\mathbf{I}_{s}[l]=\left|\mathbf{u}_{s}[l]\right|^{2}$ is the intensity of the wavefront at pixel $l$. We have then

$$
\mathbf{I}_{s}=\mathbf{B}_{o}^{2}+\mathbf{A}_{r}^{2}+2 \mathbf{B}_{o} \mathbf{A}_{r} \cos \left(\boldsymbol{\varphi}_{o}+\varphi_{r_{s}}\right)
$$

The parameter $\chi$ in (4) is a scaling factor, which can be interpreted as an exposure time (and/or as a sensitivity of the sensor). We recall that the mean and the variance of Poisson random variable $\mathbf{Y}_{s}[l]$ are equal and given by $\mathbf{I}_{s}[l] \chi$, i.e., $E\left\{\mathbf{Y}_{s}[l]\right\}=\operatorname{var}\left\{\mathbf{Y}_{s}[l]\right\}=\mathbf{I}_{s}[l] \chi$. Defining the observation signal-to-noise ratio as the ratio between the square of the mean and 
the variance of $\mathbf{Y}_{s}[l]$, we have $E^{2}\left\{\mathbf{Y}_{s}[l]\right\} / \operatorname{var}\left\{\mathbf{Y}_{s}[l]\right\}=\mathbf{I}_{s}[l] \chi$. Thus, the noise level approaches infinite when $\chi \rightarrow 0$ and approaches zero when $\chi \rightarrow \infty$. The latter case corresponds to the noiseless case and $\mathbf{Y}_{s}[l] / \chi \rightarrow \mathbf{I}_{s}[l]$ with the probability 1.

This paper is focused on the reconstruction of the object absolute phase $\boldsymbol{\varphi}_{o, a b s}$ and the amplitude $\mathbf{B}_{o}$ from the observations ${ }^{1}\left\{\mathbf{Y}_{s}, s=1, \ldots, L\right\}$. The standard approach consists in estimating phase $\varphi_{o}$ and amplitude $\mathbf{B}_{o}$ and then from $\varphi_{o}$ estimating $\varphi_{o, a b s}$ via phase unwrapping. Regarding the estimation of $\boldsymbol{\varphi}_{o}$ and $\mathbf{B}_{o}$, the intensities $\mathbf{I}_{s}$ in Eq.(5) are replaced by the observations $\mathbf{Y}_{s}$ and the obtained redundant equations are solved with respect the unknowns $\varphi_{o}$ and the amplitude $\mathbf{B}_{o}$ using the least squares method. The solutions for various configurations of the reference phase $\varphi_{r_{s}}$, for $s=1, \ldots, L$, can be found, for example, in [1], page 247. For the Poissonian observations modeled by Eq.(4), the observations $\mathbf{Y}_{s}$ are replaced with $\mathbf{Y}_{s} / \chi$ to account for the scaling parameter $\chi$. In particular, for $L=4$ and $\varphi_{r_{s}}$ taking values in the set $\{0, \pi / 2, \pi, 3 \pi / 2\}$, the least squares formulation for the phase and the amplitude of the object is

$$
\left(\widetilde{\boldsymbol{\varphi}}_{o}, \widetilde{\mathbf{B}}_{o}\right)=\arg \min _{\boldsymbol{\varphi}_{o}, \mathbf{B}_{o}} \sum_{s=1}^{4}\left\|\mathbf{Y}_{s} / \chi-\mathbf{B}_{o}^{2}+\mathbf{A}_{r}^{2}+2 \mathbf{B}_{o} \mathbf{A}_{r} \cos \left(\boldsymbol{\varphi}_{o}+\varphi_{r_{s}}\right)\right\|_{2}^{2}
$$

yielding the solution

$$
\tan \left(\widetilde{\boldsymbol{\varphi}}_{o}\right)=\frac{\mathbf{Y}_{4}-\mathbf{Y}_{2}}{\mathbf{Y}_{1}-\mathbf{Y}_{3}}, \quad \widetilde{\mathbf{B}}_{o}=\sqrt{\sum_{s=1}^{4} \mathbf{Y}_{s} / 4 \chi-\mathbf{A}_{r}^{2}} .
$$

As illustrated in the following sections, the solution (6) is highly sensitive to the counting noise present in the observations $\mathbf{Y}_{s}$. The straightforward idea is to filter somehow $\widetilde{\varphi}_{o}$, and $\widetilde{\mathbf{B}}_{o}$. This is not however a trivial procedure because the noise in the estimates $\left(\widetilde{\boldsymbol{\varphi}}_{o}, \widetilde{\mathbf{B}}_{o}\right)$ is not additive, and, what is even worse, it exhibits a complex signal dependent structure.

\footnotetext{
${ }^{1}$ To lighten the presentation, the symbols $\mathbf{Y}_{s}, s=1, \ldots, L$ are used both as random vectors and as sample vectors. The meaning should be clear from the context.
} 
We show that the iterative algorithm developed in this paper, which models the observations in an accurate way, yields precise results even for highly noisy data. Comparison of these results versus those obtained by the straightforward filtering of $\left(\widetilde{\boldsymbol{\varphi}}_{o}, \widetilde{\mathbf{B}}_{o}\right)$ is always in favor of the former.

\section{Wavefront reconstruction}

We consider the problem of wavefront reconstruction as the estimation of $\left(\mathbf{B}_{o}, \boldsymbol{\varphi}_{o, a b s}\right)$ from observations $\mathbf{Y}_{s}$, for $s=1 \ldots, L$, assuming that $\mathbf{A}_{r}$ and $\boldsymbol{\varphi}_{r_{s}}$ are given. This problem is rather challenging mainly due the periodic nature of the likelihood function with respect to the phase $\varphi_{o}$. Herein, we adopt a multiobjective optimization [7], [10] to estimate the $\left(\mathbf{B}_{o}, \varphi_{o, a b s}\right)$. The main objective of this approach is the simultaneous minimization of the negative log-likelihood function of observations given by $\sum_{s=1}^{L}-\ln p\left(\mathbf{Y}_{s} \mid \mathbf{B}_{o}, \boldsymbol{\varphi}_{o, a b s}\right)$ and of the $l_{0}$-norms of the amplitude and phase spectra modeling the sparsity of $\mathbf{B}_{o}$ and of $\boldsymbol{\varphi}_{o, a b s}$, given by $\left\|\boldsymbol{\theta}_{a, o}\right\|_{0}$ and $\left\|\boldsymbol{\theta}_{\varphi, 0}\right\|_{0}$, respectively. However, the straightforward approach based on these ideas yields complex calculations with respect to $\left(\mathbf{B}_{o}, \boldsymbol{\varphi}_{o, a b s}\right)$.

In order to make the problem manageable, we introduce auxiliary variables $\mathbf{v}_{s}$ approximating the wavefront $\mathbf{u}_{s}$ and allowing to split the optimization with respect to $\left(\mathbf{B}_{o}, \boldsymbol{\varphi}_{o}\right)$ into simpler decoupled optimizations. In addition to the negative log-likelihood function and to the $l_{0}$-norms terms, we introduce a third objective function defining the deviation between $\mathbf{v}_{s}$ and $\mathbf{u}_{s}$. The developed algorithm is iterative and composed of three successive stages each one minimizing one of the above mentioned objective functions. These successive steps are introduced in the following subsections. 


\section{A. Stage 1 : Observation noise suppression}

Let $\mathbf{v}_{s}, s=1, \ldots, L$, be approximations (estimates) of the wave fields $\mathbf{u}_{s}$ (Eq.(3)). Then, an improved approximation of $\mathbf{u}_{s}$ is obtained from the following minimization:

$$
\begin{aligned}
\widehat{\mathbf{u}}_{s} & =\arg \min _{\mathbf{u}_{s}} \mathcal{L}_{1}\left(\mathbf{u}_{s}, \mathbf{v}_{s}\right) \\
\mathcal{L}_{1}\left(\mathbf{u}_{s}, \mathbf{v}_{s}\right) & =\sum_{s=1}^{L} \sum_{l}\left[\left|\mathbf{u}_{s}[l]\right|^{2} \chi-\mathbf{Y}_{s}[l] \log \left(\left|\mathbf{u}_{s}[l]\right|^{2} \chi\right)\right]+\frac{1}{\gamma_{1}} \sum_{s=1}^{L}\left\|\mathbf{u}_{s}-\mathbf{v}_{s}\right\|_{2}^{2} .
\end{aligned}
$$

The first summand in $\mathcal{L}_{1}$ is the negative loglikelihood of Poissonian distributions (4). The second summand is a quadratic regularization (penalization) term with the weight $1 / \gamma_{1}$ defined by the difference between $\mathbf{u}_{s}$ in question and its approximation $\mathbf{v}_{s}$.

The minimization of $\mathcal{L}_{1}$ with respect to complex-valued $\mathbf{u}_{s}[l]$ gives the solution

$$
\widehat{\mathbf{u}}_{s}[l]=\mathbf{b}_{s}[l] \exp \left(j \cdot \operatorname{angle}\left(\mathbf{v}_{s}[l]\right)\right.
$$

where

$$
\mathbf{b}_{s}[l]=\frac{\left|\mathbf{v}_{s}[l]\right| /\left(\gamma_{1} \chi\right)+\sqrt{\left|\mathbf{v}_{s}[l]\right|^{2} /\left(\gamma_{1} \chi\right)^{2}+4 \mathbf{Y}_{s}[l]\left(1+1 /\left(\gamma_{1} \chi\right)\right) / \chi}}{2\left(1+1 /\left(\gamma_{1} \chi\right)\right)}
$$

Note that for $\gamma_{1} \chi \rightarrow \infty$ (noiseless case), we have

$$
\widehat{\mathbf{u}}_{s}[l] \rightarrow \sqrt{\mathbf{Y}_{s}[l] / \chi} \exp \left(j \cdot \operatorname{angle}\left(\mathbf{v}_{s}[l]\right), \quad s=1, \ldots, L\right.
$$

This solution coincides with the famous Gerchberg-Saxton-Fienup rule, where the amplitude of the estimate is replaced by the square root of the intensity measurement and the phase is equal to its predicted value. We conclude that the widely used Gerchberg-Saxton-Fienup rule is an approximation of the maximum likelihood estimate of $\mathbf{u}_{s}$ only valid for in high signal-to-noise ratio scenarios. 


\section{B. Stage 2 : Parametrization of object wavefront}

The variables $\mathbf{u}_{s}$ are represented in Eq.(3) as a function of $\left(\boldsymbol{\varphi}_{o}, \mathbf{B}_{o}\right)$. Let us assume that we observe an $\mathbf{u}_{s}+\varepsilon$, where $\varepsilon$ is an additive perturbation. From these observations, we infer $\left(\boldsymbol{\varphi}_{o}, \mathbf{B}_{o}\right)$ by solving the optimization

$$
\begin{aligned}
\left(\widehat{\boldsymbol{\varphi}}_{o}, \widehat{\mathbf{B}}_{o}\right) & =\arg \min _{\boldsymbol{\varphi}_{o}, \mathbf{B}_{o}} \mathcal{L}_{2}\left(\mathbf{B}_{o}, \boldsymbol{\varphi}_{o}, \mathbf{A}_{r}, \mathbf{u}_{s}\right) \\
\mathcal{L}_{2}\left(\mathbf{B}_{o}, \boldsymbol{\varphi}_{o}, A_{r}, \mathbf{u}_{s}\right) & =\sum_{s=1}^{L}\left\|\mathbf{u}_{s}-\left(\mathbf{B}_{o} \exp \left(j \boldsymbol{\varphi}_{o}\right)+\mathbf{A}_{r} \exp \left(-j \varphi_{r_{s}}\right)\right)\right\|_{2}^{2}
\end{aligned}
$$

The minimization of $\mathcal{L}_{2}$ with respect to $\boldsymbol{\varphi}_{o}$ and $\mathbf{B}_{o}$ gives

$$
\widehat{\boldsymbol{\varphi}}_{o}=\operatorname{angle}\left(\sum_{s=1}^{L} \mathbf{u}_{s}\right), \quad \widehat{\mathbf{B}}_{o}=\operatorname{Re}\left(\exp \left(-j \boldsymbol{\varphi}_{o}\right) \sum_{s} \mathbf{u}_{s}\right) / 4
$$

\section{C. Stage 3: Sparsification (filtering) of phase and amplitude}

As it was already discussed in Section 2, we use the separate sparse modeling for the absolute phase $\boldsymbol{\varphi}_{o, a b s}$ and amplitude $\mathbf{B}_{o}$ of the wavefront $\mathbf{u}_{o} \equiv \mathbf{B}_{o} \exp \left(j \boldsymbol{\varphi}_{o}\right)$. The corresponding sparsity criterion is similar to the one introduced in [7]:

$$
\begin{aligned}
\left(\widehat{\boldsymbol{\theta}}_{\varphi_{o}}, \widehat{\boldsymbol{\theta}}_{B_{o}}\right)= & \arg \min _{\boldsymbol{\theta}_{\varphi_{o}}, \boldsymbol{\theta}_{B_{o}}} \mathcal{L}_{3}\left(\boldsymbol{\theta}_{\varphi_{o}}, \boldsymbol{\theta}_{B_{o}}, \boldsymbol{\varphi}_{o, a b s}, \mathbf{B}_{o}\right), \\
\mathcal{L}_{3}\left(\boldsymbol{\theta}_{\varphi_{o}}, \boldsymbol{\theta}_{B_{o}}, \boldsymbol{\varphi}_{o, a b s}, \mathbf{B}_{o}\right)= & \tau_{a} \cdot\left\|\boldsymbol{\theta}_{B_{o}}\right\|_{0}+\tau_{\varphi} \cdot\left\|\boldsymbol{\theta}_{\varphi_{o}}\right\|_{0} \\
& \left.+\frac{1}{2} \| \boldsymbol{\theta}_{B_{o}}-\boldsymbol{\Phi}_{a, o} \mathbf{B}_{o}\right)\left\|_{2}^{2}+\frac{1}{2}\right\| \boldsymbol{\theta}_{\varphi_{o}}-\boldsymbol{\Phi}_{\varphi, o} \boldsymbol{\varphi}_{o, a b s} \|_{2}^{2} .
\end{aligned}
$$

The presence of the $l_{0}$-norms in (15) enforces sparsity on the phase spectrum $\boldsymbol{\theta}_{\varphi_{o}}$ and on the amplitude spectrum $\boldsymbol{\theta}_{B_{o}}$. The regularization terms $\left.\frac{1}{2} \| \boldsymbol{\theta}_{B_{o}}-\boldsymbol{\Phi}_{B_{o}} \mathbf{B}_{o}\right) \|_{2}^{2}$ and $\frac{1}{2} \| \boldsymbol{\theta}_{\varphi_{o}}-$ $\boldsymbol{\Phi}_{\varphi_{o}} \boldsymbol{\varphi}_{o, a b s} \|_{2}^{2}$ are defined by the predictions $\boldsymbol{\Phi}_{B_{o}} \mathbf{B}_{o}$ and $\boldsymbol{\Phi}_{\varphi_{o}} \boldsymbol{\varphi}_{o, a b s}$ for the amplitude and phase spectra $\boldsymbol{\theta}_{B_{o}}$ and $\boldsymbol{\theta}_{\varphi_{o}}$, respectively.

The minimization of $\mathcal{L}_{3}$ with respect to the spectra $\boldsymbol{\theta}_{\varphi_{o}}, \boldsymbol{\theta}_{B_{o}}$ gives the well known hard- 
thresholding solutions

$$
\begin{aligned}
& \widehat{\boldsymbol{\theta}}_{B_{o}}=\boldsymbol{\Phi}_{a, o} \mathbf{B}_{o} \cdot H\left(\left|\boldsymbol{\Phi}_{B_{o}} \mathbf{B}_{o}\right|-T h_{a}\right) \\
& \widehat{\boldsymbol{\theta}}_{\varphi_{o}}=\boldsymbol{\Phi}_{\varphi, o} \boldsymbol{\varphi}_{o} \cdot H\left(\left|\boldsymbol{\Phi}_{\varphi_{o}} \boldsymbol{\varphi}_{o, a b s}\right|-T h_{\varphi}\right)
\end{aligned}
$$

where $T h_{a}=\sqrt{2 \tau_{a}}$ and $T h_{\varphi}=\sqrt{2 \tau_{\varphi}}$ are thresholds for the amplitude and the phase respectively, and $H$ is the Heaviside step function. The spectral coefficients $\left|\boldsymbol{\Phi}_{\varphi_{o}} \boldsymbol{\varphi}_{o, a b s}\right|$ and $\left|\mathbf{\Phi}_{B_{o}} \mathbf{B}_{o}\right|$ smaller than the corresponding thresholds are zeroed in Eq. (16).

Using these estimates, we define the auxiliary variable $\mathbf{v}_{s}$ as the approximation of $\mathbf{u}_{o}$ in the form:

$$
\mathbf{v}_{s}=\left(\boldsymbol{\Psi}_{\boldsymbol{\theta}_{B_{o}}} \widehat{\boldsymbol{\theta}}_{B_{o}}\right) \exp \left(j \Psi_{\boldsymbol{\varphi}_{o}} \widehat{\boldsymbol{\theta}}_{\boldsymbol{\varphi}_{o}}\right)+\mathbf{A}_{r} \exp \left(-j \varphi_{r_{s}}\right)
$$

The variable $\mathbf{v}_{s}$ in Eq.(7) corresponds to a quadratic splitting introduced in $\mathcal{L}_{1}\left(\mathbf{u}_{s}, \mathbf{v}_{s}\right)$ to decompose this likelihood criterion and the sparsity criterion $\mathcal{L}_{3}\left(\boldsymbol{\theta}_{\varphi_{o}}, \boldsymbol{\theta}_{B_{o}}, \boldsymbol{\varphi}_{o, a b s}, \mathbf{B}_{o}\right)$ in two more manageable objective functions. Therefore, the objective function $\mathcal{L}_{1}\left(\mathbf{u}_{s}, \mathbf{v}_{s}\right)$ jointly with $\mathcal{L}_{2}\left(\mathbf{B}_{o}, \boldsymbol{\varphi}_{o}, \mathbf{A}_{r}, \mathbf{u}_{s}\right)$ are interpretable as the negative Poissonian loglikelihood function, and the objective function $\mathcal{L}_{3}\left(\boldsymbol{\theta}_{\varphi_{o}}, \boldsymbol{\theta}_{B_{o}}, \boldsymbol{\varphi}_{o, a b s}, \mathbf{B}_{o}\right)$ is a weighted measure between the sparsity of $\mathbf{B}_{o}$ and $\boldsymbol{\varphi}_{o}$ and their representation errors, using the analysis frames $\boldsymbol{\Phi}_{B_{o}}$ and $\boldsymbol{\Phi}_{\varphi_{o}}$. We conclude therefore that the multiobjective optimization (7), (11), (14) aims at finding object $\mathbf{u}_{o}$ which is simultaneously likely to have produced the observation and to have sparse representation.

Naturally, the success of the sparse imaging depends on how reach and redundant are the dictionaries used for the analysis and the synthesis. In our experiments for the analysis and synthesis operations, we use the BM3D frames, where BM3D is an abbreviation for BlockMatching and 3D filtering [10]. Let us recall basic ideas of this advanced technique. At the first stage phase and amplitude of the wavefront are partitioned into small overlapping square 
patches. For each patch a group of similar patches is collected which are stacked together and form a 3D array separately for amplitude and phase. This stage is called grouping. The entire 3D arrays are projected onto a 3D transform orthonormal basis. The obtained spectral coefficients are hard-thresholded and inverse 3D transform gives filtered patches, which are returned to the original positions of these patches of the phase and amplitude images, respectively. This stage is called collaborative filtering. The process is repeated for all pixels of the entire wavefront. The final estimates of the amplitude and the phase are given by calculating weighted means of the estimates obtained for common pixels of the overlapped filtered patches of the amplitude and the phase. This last stage is called aggregation. The details of BM3D as an advanced image filter can be seen in [11].

In our technique, as it is implemented in this paper, grouping, analysis, synthesis, and hard-thresholding are combined in a single procedure which we call BM3D. This procedure in parallel and independently is applied to amplitude and phase variables. Then, the solutions (16) can be presented in the following short form:

$$
\begin{aligned}
& \left(\boldsymbol{\Psi}_{\boldsymbol{\theta}_{B_{o}}} \widehat{\boldsymbol{\theta}}_{B_{o}}\right)=\mathrm{BM} 3 \mathrm{D}_{\mathrm{ampl}}\left(\mathbf{B}_{o}, T h_{a}\right),
\end{aligned}
$$

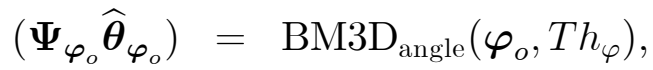

where BM3D denotes algorithm applied to the amplitude and to the phase with different indexes just because different parameters of the algorithm can be used for the amplitude and the phase. 


\section{Algorithm}

Combining the solutions obtain in Section 4, we arrive to the following Sparse $\mathbf{P h a s e}$ and Amplitude Reconstruction (SPAR) algorithm.

\section{SPAR (Sparse Phase Amplitude Reconstruction) algorithm}

Input: $\left\{\mathbf{Y}_{s}\right\}, s=1, \ldots, L$;

Output: $\hat{\varphi}_{o, a b s}^{T}, \hat{\mathbf{B}}_{o}^{T}$;

Initialization: $t=0, \hat{\varphi}_{o}^{0}, \hat{\mathbf{B}}_{o}^{0}$;

$$
\mathbf{v}_{s}^{0}=\hat{\mathbf{B}}_{o}^{0} \exp \left(j \hat{\boldsymbol{\varphi}}_{o}^{0}\right)+\mathbf{A}_{r} \exp \left(-j \varphi_{r_{s}}\right), s=1, \ldots, L
$$

Repeat for $t=0,1, \ldots$,

1. Poissonian noise suppression (Eq. (8)):

$\hat{\mathbf{u}}_{s}^{t}=\hat{\mathbf{B}}_{s}^{t} \exp \left(j \cdot \operatorname{angle}\left(\mathbf{v}_{s}^{t}\right), s=1, \ldots, L, \hat{\mathbf{B}}_{s}^{t}=\left(\hat{\mathbf{b}}_{s}^{t}[l]\right)\right.$

2. Parametrization of $\hat{\mathbf{u}}_{o}^{t}$ (Eq. (13)):

$$
\widehat{\boldsymbol{\varphi}}_{o}^{t}=\operatorname{angle}\left(\sum_{s=1}^{L} \mathbf{u}_{s}^{t}\right), \widehat{\mathbf{B}}_{o}^{t}=\operatorname{Re}\left(\exp \left(-j \widehat{\boldsymbol{\varphi}}_{o}^{t}\right) \sum_{s} \mathbf{u}_{s}^{t}\right) / 4
$$

3. Phase unwrapping (PUMA [12]):

$$
\hat{\varphi}_{o, a b s}^{t}=\mathcal{W}^{-1}\left(\hat{\varphi}_{o}^{t}\right)
$$

4. Sparsification (filtering) of amplitude and absolute phase (Eqs. (18)):

$$
\begin{gathered}
\hat{\boldsymbol{\varphi}}_{o, a b s}^{t+1}=B M 3 D_{\text {angle }}\left(\hat{\boldsymbol{\varphi}}_{o, a b s}^{t}, T h_{\varphi}\right), \\
\hat{\mathbf{B}}_{o}^{t+1}=B M 3 D_{\text {ampl }}\left(\hat{\mathbf{B}}_{o}^{t}, T h_{a}\right) ;
\end{gathered}
$$

5. Updating $\mathbf{v}_{s}^{t}$ :

$$
\mathbf{v}_{s}^{t+1}=\mathbf{B}_{o}^{t+1} \exp \left(j \hat{\boldsymbol{\varphi}}_{o, a b s}^{t+1}\right)+\mathbf{A}_{r} \exp \left(-j \varphi_{r_{s}}\right), s=1, \ldots, L
$$

6. $t=t+1$;

Until stopping criterion is satisfied. 
The SPAR algorithm is initialized by $\mathbf{v}_{s}^{0}, s=1, \ldots, L$, where $\mathbf{A}_{r}$ is assumed known and $\hat{\boldsymbol{\varphi}}_{o, a b s}^{0}, \hat{\mathbf{B}}_{o}^{0}$ are obtained by filtering of the raw data $\tilde{\boldsymbol{\varphi}}_{o}^{0}, \tilde{\mathbf{B}}_{o}^{0}$ calculated according to Eqs.(6). As it is mentioned in Section 3, these latter estimates can be quite noisy. In our experiments for this filtering, we use $\mathrm{BM} 3 \mathrm{D}_{\bmod }$ for $\mathbf{B}$, which gives $\hat{\mathbf{B}}_{o}^{0}=B M 3 D_{\text {ampl }}\left(\widetilde{\mathbf{B}}_{o}\right)$, and the WFT (windowed Fourier transform) algorithm [13], [14] for filtering of the wrapped phase $\widetilde{\boldsymbol{\varphi}}_{o}$, $\widehat{\boldsymbol{\varphi}}_{o}^{0}=\mathrm{WFT}\left(\widetilde{\boldsymbol{\varphi}}_{o}\right)$. Both algorithms, BM3D $\mathrm{D}_{\mathrm{mod}}$ and WFT, yield state-of-the-art results in the corresponding fields for the image and the wrapped phase denoising, respectively.

At Step 1 the noise in the magnitudes of $\widehat{\mathbf{u}}_{s}^{t}, s=1, \ldots, L$, is filtered. The level of the noise suppression is defined by the parameter $\gamma_{1}$. For large values of $\gamma_{1}$ the amplitudes are defined by the observed $\mathbf{Y}_{s}$, i.e., no filtering (see Eq. (9)). The phase of $\widehat{\mathbf{u}}_{s}^{t}$ is equal to the phase of $\widehat{\mathbf{v}}_{s}^{t}$ for any $\gamma_{1}$.

At Step 2 the object wavefront $\widehat{\mathbf{u}}_{o}^{t}$ is defined. For phase unwrapping at Step 3 we use the state-of-the-art PUMA algorithm [12], which is able to cope with piecewise smooth surfaces of the absolute phase. The phase and the amplitude of the object wave front are filtered at Step 4 by BM3D filters. At Step 5 the variable $\mathbf{v}_{s}$, i.e., the estimate of the wave front impinging on the sensor, is updated. In our experiments we use a fixed number, $T$, of iterations as a stopping criterion. Thus, the output of the algorithm are defined by the variables $\hat{\varphi}_{o, a b s}^{T}, \mathbf{B}_{o}^{T}$.

The block-scheme of this algorithm are shown in Fig. 2.

\section{Experiments}

The experiments presented in this section are obtained for a quadratic amplitude and three types of a phase surface: Gaussian, Truncated Gaussian, and Shear Plane, exploited in [7]. The ratio maximum-to-minimum values of the amplitude is equal to 2 with the maximum value at the central point of the image. This setting models wavefronts with non-uniform 
intensities.

The accuracy of the wavefront reconstruction is characterized by ISNR (improvement in signal-to-noise ratio in $\mathrm{dB}$ ) independently for the amplitude and exponential complex components of the wavefront and by RMSE (root mean square error) for the absolute phase:

$$
\begin{aligned}
& \operatorname{ISNR}_{a m p}=10 \log _{10} \frac{\left\|\widetilde{\mathbf{B}}_{o}-\mathbf{B}_{o}\right\|_{2}^{2}}{\left\|\widehat{\mathbf{B}}_{o}^{50}-\mathbf{B}_{o}\right\|_{2}^{2}}, \\
& \mathrm{ISNR}_{\exp }=10 \log _{10} \frac{\left\|\exp \left(j \widetilde{\boldsymbol{\varphi}}_{o}\right)-\exp (j \boldsymbol{\varphi})\right\|_{2}^{2}}{\left\|\exp \left(j \widehat{\boldsymbol{\varphi}}_{o}^{50}\right)-\exp (j \boldsymbol{\varphi})\right\|_{2}^{2}}, \\
& \mathrm{ISNR}_{\text {phase }, \text { wrap }}=10 \log _{10} \frac{\left\|\mathcal{W}\left(\mathcal{W}\left(\widetilde{\boldsymbol{\varphi}}_{o}\right)-\boldsymbol{\varphi}_{o}\right)\right\|_{2}^{2}}{\left\|\mathcal{W}\left(\mathcal{W}\left(\widehat{\boldsymbol{\varphi}}_{o}^{50}\right)-\boldsymbol{\varphi}_{o}\right)\right\|_{2}^{2}}, \\
& \operatorname{RMSE}_{\text {phase }, a b s}=\frac{1}{N}\left\|\widehat{\boldsymbol{\varphi}}_{o, a b s}^{50}-\boldsymbol{\varphi}_{o}\right\|_{2}^{2} .
\end{aligned}
$$

Using the complex exponent in $\mathrm{ISNR}_{\exp }$ allows simultaneously estimating the accuracy of the real and imaginary parts of the wavefront and in this way to evaluate the accuracy of the wavefront reconstruction overall. The amplitude $\mathbf{B}_{o}$ can be included in this criteria but we prefer to evaluate the accuracy separately for the phase and the amplitude because the phase inference in many application is the main objective. Note that in all cases we calculate ISNR with respect to the observed noisy raw data $\widetilde{\boldsymbol{\varphi}}_{o}$ and $\widetilde{\mathbf{B}}_{o}$. The filtered values of these variables, i.e., $\widehat{\varphi}_{o}^{0}$ and $\widehat{\mathbf{B}}_{o}^{0}$, are used for initialization of the algorithm.

\section{A. Visual comparison of the reconstructions}

The experiments herein conducted are produced for various values of the parameter $\chi$ defining the amount of noise in observations. We are mostly concerned with highly noisy scenarios, where, as seen below, SPAR exhibits the strongest advantage compared with the competitors. The essential accuracy improvement is observed also for the low-level noise, when visually this improvement cannot be noticed.

Figs. 3 to 6 show the reconstruction results for the Gaussian phase, with 44 radians of phase 
range and the quadratic amplitude. The scale parameter controlling the level of the noise is set to $\chi=0.05$. We can see that the observations (raw data) $\widetilde{\varphi}_{o}$ (Fig.3a) and $\widetilde{\mathbf{B}}_{o}$ (Fig.5a) obtained according to Eq.(6) are indeed extremely noisy. Fig.3b and Fig.5b show $\widehat{\varphi}_{o}^{0}$ and $\widehat{\mathbf{B}}_{o}^{0}$ obtained by pre-filtering of these noisy data as $\widehat{\boldsymbol{\varphi}}_{o}^{0}=\mathrm{WFT}\left(\widetilde{\boldsymbol{\varphi}}_{o}\right)$ and $\widehat{\mathbf{B}}_{o}^{0}=\mathrm{BM} 3 \mathrm{D}_{\text {ampl }}\left(\widetilde{\mathbf{B}}_{o}\right)$. Fig.3c and Fig.5c illustrate SPAR performance: the obtained estimates are very close the true wrapped phase and the amplitude shown in Fig.3d and Fig.5d, respectively.

Fig.4 presents the corresponding results for the unwrapped phase, respectively: the initial

reconstruction $\widehat{\varphi}_{o, a b s}^{0}$ (Fig. 4a), the SPAR reconstruction $\widehat{\varphi}_{o, a b s}^{50}$ (Fig.4b) and the true absolute phase (Fig.4c). In the last image of this series (Fig.6), we show the SPAR performance as a function of the number of iterations. The convergence for the phase and amplitude ISNR is reached, from a practical point of view, in 50 iterations.

The next two images, shown in Fig.7 and Fig.8, show similar results for the truncated Gaussian phase, the quadratic amplitude, and $\chi=0.25$. We show only the images concerning the phase because the images for the amplitude and the dependence of the accuracy on iterations are quite similar to shown in Fig.5-Fig.6. We can see quite impressive results where the noise effects are suppressed nearly completely.

The phase results for the shear plane phase are shown in the two images (Fig.9 and Fig.10). Again we can see quite accurate reconstruction of this linearly varying absolute phase covering the range 79 radians. In all images, we can note that the numerical accuracy improvement with respect to the initial estimates is very impressive.

\section{B. Parameters of the SPAR algorithm}

The noise parameter $\chi$ takes values in the interval $[0.05,5]$. For $\chi_{\max }=5.0$, the data are practically noiseless, while for $\chi_{\min }=0.05$ the noise level is so high that an acceptable 
quality reconstruction of the absolute phase from $\widehat{\varphi}_{o}^{0}$, using the PUMA algorithm, becomes impossible.

As expected, SPAR performance depends on the parameters of the algorithm. We have kept BM3D parameters for synthesis and analysis frames fixed in all our experiments. The size of the image patches is always $8 \times 8$ and the group size is limited by the number 25 . The step size between the neighboring patches is equal to 2 .

The parameters $\gamma_{1}$ appearing in $\mathcal{L}_{1}$ and the thresholds $T h_{\varphi}$ and $T h_{a}$, used in Eqs.(16) and (17), respectively, are set heuristically as follows: $\gamma_{1}=0.4 ; T h_{a}=0.02 ; T h_{\varphi}=$ $0.02 / \sqrt{\chi / \chi_{\min }}$. Thus, the phase threshold is taken depending on $\chi$, larger $\chi$ results in a smaller value of the phase threshold.

For the truncated Gaussian phase, we have used $\chi=0.25$ because for lower values of $\chi$ the unwrapping gives poor results owing to high level of the noise present in the wrapped phases $\widetilde{\varphi}_{o}$ and $\widehat{\varphi}_{o}^{0}$.

\section{C. Numerical evaluation of reconstruction accuracy}

A detail picture of the accuracy achieved by the SPAR algorithm can be seen in Table 1. Here we show results for different level of noise by varying $\chi$ from $\chi_{\min }=0.05$ to $\chi_{\max }=5.0$.

The three numbers shown for each criteria regards, from the left to the right, a) SPAR algorithm, b) initial estimates $\widehat{\boldsymbol{\varphi}}_{o}^{0}$ and $\widehat{\mathbf{B}}_{o}^{0}$, making evident how far the initial estimate is improved by SPAR, c) best estimates (shown in brackets) which can be achieved using the separate filtering of $\widetilde{\boldsymbol{\varphi}}_{o}, \widetilde{\mathbf{B}}_{o}$ given by Eq.(6).

Concerning these best estimates we wish to note the following. The estimate for the amplitude is obtained by $\mathrm{BM} \mathrm{D}_{a m p}$ optimized with respect to the threshold parameter $T h_{a}$ and this optimization is produced separately for each experiment. In a similar way, in order to 
obtain the best estimate for the phase, we use the WFT algorithm. Remind that WFT is derived for the exponential observation model $\mathbf{u}_{s}=\mathbf{B}_{o} \exp \left(j \boldsymbol{\varphi}_{o}\right)+\sigma\left(\varepsilon_{1, s}+j \varepsilon_{2, s}\right)$, where $\boldsymbol{\varepsilon}_{1, s}$ and $\varepsilon_{2, s}$ are independent Gaussian, zero mean with the standard deviations equal to 1 . The standard deviation $\sigma$ of this complex-valued noise is an important parameter of WFT: larger values of $\sigma$ result in stronger smoothing and smaller values result in noisier estimates. The optimization of WFT is produced with respect to $\sigma$. In this way we obtain the so-called "oracle" estimates assuming that the true amplitude and phase are given in advance and

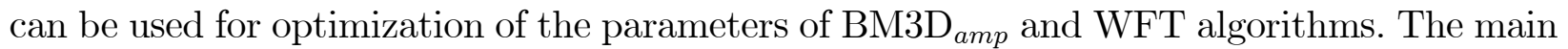
idea behind this modeling is providing clear evidences that even with the optimal (but impractical) conditions, the separate filtering of the raw observations $\widetilde{\boldsymbol{\varphi}}_{o}, \widetilde{\mathbf{B}}_{o}$ given by Eq.(6) cannot competes with the results of the SPAR algorithm.

We can see in Table 1 for the criteria $\mathrm{ISNR}_{\text {phase,wrap }}, \mathrm{ISNR}_{\text {exp }}$ and $\mathrm{ISNR}_{\text {ampl }}$ that these oracle estimates are always better than the initial phase and amplitude estimates. However, this optimal tuning of WFT is not always good for the absolute phase reconstruction if the data are very noisy. In particular, for the Gaussian phase, $\chi=0.05$, we can see that $\mathrm{RMSE}_{\text {phase,abs }}=1.72$ for the optimized WFT, while the initial estimate shows $\mathrm{RMSE}_{\text {phase }, a b s}=0.46$. Similarly, for the truncated Gaussian phase $(\chi=.25$ and $\chi=0.5)$ we can see for the optimized WFT estimate $\mathrm{RMSE}_{\text {phase,abs }}=1.82$ and $\mathrm{RMSE}_{\text {phase }, a b s}=1.64$ versus $\mathrm{RMSE}_{\text {phase }, a b s}=1.07$ and $\mathrm{RMSE}_{\text {phase }, a b s}=.02$, respectively, obtained by the initial phase estimate $\widehat{\varphi}_{o}^{0}=\mathrm{WFT}\left(\widetilde{\varphi}_{o}\right)$.

These large values of $\mathrm{RMSE}_{\text {phase,abs }}$ mean that the absolute phase reconstruction is seriously different from the true absolute phase. For initialization of SPAR, we use WFT with significantly lower values of $\sigma$, than those appeared in the optimized oracle estimates, which 
have been optimized for estimation of the interferometric wrapped phase. As a result, the initial estimate of the wrapped phase given by WFT is noisier than that obtained by the optimized oracle one but the essential features of the wrapped phase are preserved. The phase unwrapping is very delicate procedure which is sensitive to even small errors in the corresponding wrapped phase. The optimization of WFT for the wrapped phase results in a compromise between the noise variance and the bias and later can be strong enough in order to destroy the structure of the wrapped phase essential for unwrapping.

The Poissonian noise can be characterized in terms of the mean signal-to-noise ratio per pixel $(m S N R p)$ and the mean value of photons per pixel $\left(N_{\text {photon }}\right)$. The former criterion for the Poissonian observations is calculated for each experiments as $\operatorname{10log}_{10}(\operatorname{mean}(Y) / \sqrt{\operatorname{mean}(Y)})$, mean $(Y)=\frac{1}{4 N} \sum_{l=1}^{N} \sum_{s}^{4} Y_{s}[l]$. In particular, for the Gaussian phase we found: $\chi=0.05$ corresponds to $m S N R p=2.8 \mathrm{~dB}$ and $N_{\text {photon }}=3.6$; $\chi=0.5$ corresponds to $m S N R p=7.8 \mathrm{~dB}$ and $N_{\text {photon }}=35.8 ; \chi=5$ corresponds to $m S N R p=$ $12.8 \mathrm{~dB}$ and $N_{\text {photon }}=358$. For small values of $\chi$, many observations take zero values. For instance, for the results, shown in Fig.3-Fig.6 about $10 \%$ of the observed values of $\mathbf{Y}_{s}$ are zeros.

For our experiments, we used MATLAB R2012b and the computer with the processor Intel(R) Core(TM) 2 Duo CPU P8400@ 2.26 GHz. The complexity of the algorithm is characterized by the time required for one iteration. For image $100 \times 100$ this time is about $1 \mathrm{sec} /$ iteration.

We make our MATLAB programs for the demo version of our algorithms publicly available for testing: http://www.cs.tut.fi/ lasip/DDT/. 


\section{Conclusion}

This paper introduced a variational approach for reconstruction of wavefront, i.e., absolute phase and amplitude, from Poissonian phase-shifting measurements. Sparse modeling of amplitude and absolute phase is one of the key elements of the developed algorithm. The other key step is the adoption of multiobjective optimization, which allowed to decompose an unbearable optimization into an iterative procedure solving, in each iteration, a set of much simpler subproblems. Armed with the sparse modeling framework, and with the multiobjective optimization strategy, we introduced the sparse phase and amplitude reconstruction (SPAR) algorithm. SPAR takes into full consideration the Poissonian (photon counting) measurements, uses data adaptive BM3D frames as a sparse representation for the amplitude and for the absolute phase. SPAR effectiveness was documented by comparing its performance with that of competitors in a series of experiments, where it systematically outperformed the competitors.

\section{Acknowledgments}

This work is supported by the Academy of Finland, project no. 138207, 2011-2014, and by Portuguese Science and Technology Foundation under Projects PEst-OE/EEI/LA0008/2013 and PTDC/EEI-PRO/1470/2012.

\section{References}

1. Th. Kreis. Handbook of Holographic Interferometry. (Wiley-VCH, Berlin, 2005).

2. J. W. Goodman, Introduction to Fourier Optics, 3rd ed. (Roberts \& Company, Englewood, 2005).

3. V. Katkovnik, J. Bioucas-Dias, H. Hao, "Wavefront reconstruction from noisy fringe observations via sparse coding," The 7th International Workshop on Advanced Optical Imaging and Metrology FRINGE 2013, 8-11th September 2013, Nürtingen, Germany. 
4. M. Elad. Sparse and Redundant Representations: from Theory to Applications in Signal and Image Processing. (Springer, 2010).

5. D. C. Ghiglia and M. D. Pritt. Two-Dimensional Phase Unwrapping: Theory, Algorithms, and Software. (Wiley, 1998).

6. H. Hongxing, J. M. Bioucas-Dias, and V. Katkovnik, "Interferometric phase image estimation via sparse coding in the complex domain," IEEE Transactions on Geoscience and Remote Sensing, (2014, in print).

7. V. Katkovnik and J. Astola, " High-accuracy wavefield reconstruction: decoupled inverse imaging with sparse modeling of phase and amplitude," J. Opt. Soc. Am. A 29, pp. $44-54$ (2012).

8. V. Katkovnik and J. Astola, "Phase retrieval via spatial light modulator phase modulation in $4 \mathrm{f}$ optical setup: numerical inverse imaging with sparse regularization for phase and amplitude," J. Opt. Soc. Am. A $29,105-116(2012)$.

9. V. Katkovnik and J. Astola, "Compressive sensing computational ghost imaging," J. Opt. Soc. Am. A, vol. 29, Iss. 8, pp. 1556-1567 (2012).

10. A. Danielyan, V. Katkovnik, and K. Egiazarian, "BM3D frames and variational image deblurring," IEEE Trans. Image Process. vol. 21, pp. 1715 - 1728, (2012).

11. K. Dabov, A. Foi, V. Katkovnik, and K. Egiazarian, "Image denoising by sparse 3D transform-domain collaborative filtering", IEEE Trans. Image Process., vol. 16, no. 8, pp. 2080-2095, (2007).

12. J. M. Bioucas-Dias and G. Valadão, " Phase unwrapping via graph cuts," IEEE Transactions on Image Processing 16 (3), pp. 698-709, (2007).

13. Q. Kemao, "Two-dimensional windowed Fourier transform for fringe pattern analysis: principles, applications and implementations," Optics and Lasers in Engineering, vol. 45, no. 2, pp. $304-317$ (2007).

14. Q. Kemao. Windowed Fringe Pattern Analysis. (SPIE, Bellingham, Whashington, 2013). 


\section{List of Figure Captions}

Fig.1. Phase-shift interferometry: on-axis setup. In this Michelson configuration a single laser beam of coherent light is split into two beams by a beam splitter. The path difference traveled by the reference (mirror) beam and object beam is reflected in the intensity of the aggregated beam $\mathbf{u}_{s}$. This intensity is measured by the sensor array. Shift of the mirror is used in order to introduce different phase shifts between the object and reference beams used in order to reconstruct the object wavefront from the intensity measurements.

Fig.2. SPAR algorithm: block-scheme.

Fig.3. Wrapped phase for the Gaussian phase object, from left to right: the noisy raw data, the initial $W F T$ estimate, $S P A R$ reconstruction, true image.

Fig.4. The absolute (unwrapped) phase for the Gaussian phase object, from left to right: the initial WFT estimate, SPAR reconstruction, true image.

Fig.5. Surfaces for the quadratic amplitude, from left to right: the noisy raw data, the initial $\mathrm{BM} \mathrm{D}_{a m p l}$ estimate, $S P A R$ reconstruction, true image.

Fig.6. The accuracy of the wavefront reconstruction (object with the Gaussian phase) versus the number of iterations, from left to right: $I S N R_{\exp }$ for the exponential wavefront $\exp (j \varphi), I S N R_{a m p l}$ for the quadratic amplitude.

Fig.7. The wrapped phase for the truncated Gaussian phase object, from left to right: the noisy raw data, the initial $W F T$ estimate, $S P A R$ reconstruction, true image.

Fig.8. The absolute (unwrapped) phase for the truncated Gaussian phase object, from left to right: the initial $W F T$ estimate, $S P A R$ reconstruction, true image.

Fig.9. The wrapped phase for the shear plane phase object, from left to right: the noisy raw data, the initial $W F T$ estimate, $S P A R$ reconstruction, true image. 
Fig.10. The absolute (unwrapped) phase for shear plane phase object, from left to right: the initial $W F T$ estimate, $S P A R$ reconstruction, true image. 


\section{List of Table Captions}

Table 1. Accuracy criteria for phase and amplitude reconstruction. Each row corresponds to different phase function of the wavefront. The three numbers shown for each criteria regards, from the left to the right, SPAR, the initialization of SPAR, and the best oracle estimate provided by WFT (in brackets). 
Table 1. Accuracy criteria for phase and amplitude reconstruction. Each row corresponds to different phase function of the wavefront. The three numbers shown for each criteria regards, from the left to the right, SPAR, the initialization of SPAR, and the best oracle estimate provided by WFT (in brackets).

\begin{tabular}{||l|l|l|l|l|l||}
\hline Phase & $\chi$ & ISNR $_{\text {phase } \text { wrap }}$ & ISNR $_{\text {exp }}$ & RMSE $_{\text {phase }, a b s}$ & ISNR $_{a m p l}$ \\
\hline \hline Gauss & 0.05 & $17.6 \mid 9.47(10.6)$ & $15.9 \mid 8.54(9.78)$ & $0.178 \mid 0.46(1.72)$ & $22.3 \mid 9.43(16.9)$ \\
& 0.5 & $19.25 \mid 10.73(16.1)$ & $18.6 \mid 10.10(15.15)$ & $0.065 \mid .17(.0943)$ & $17.6 \mid 9.54(17.17)$ \\
\hline Gauss & 0.25 & $20.09 \mid 11.9(15.3)$ & $18.9 \mid 10.8(14.22)$ & $0.324 \mid 1.07(1.82)$ & $18.22 \mid 9.53(17.1)$ \\
\hline Trunc & 0.5 & $19.7 \mid 9.65(13.95)$ & $19.0 \mid 9.01(13.35)$ & $0.445 \mid 0.2(1.64)$ & $18.3 \mid 10.1(17.33)$ \\
& 2.5 & $17.7 \mid 4.78(11.87)$ & $17.6 \mid 4.74(11.82)$ & $0.030 \mid 0.1317(0.0586)$ & $16.05 \mid 8.9(15.82)$ \\
\hline \hline Shear & 0.05 & $21.5 \mid 10.5(14.77)$ & $20.5 \mid 9.15(13.28)$ & $0.238 \mid 2.14(4.76)$ & $21.3 \mid 10.3(16.93)$ \\
\hline \hline
\end{tabular}




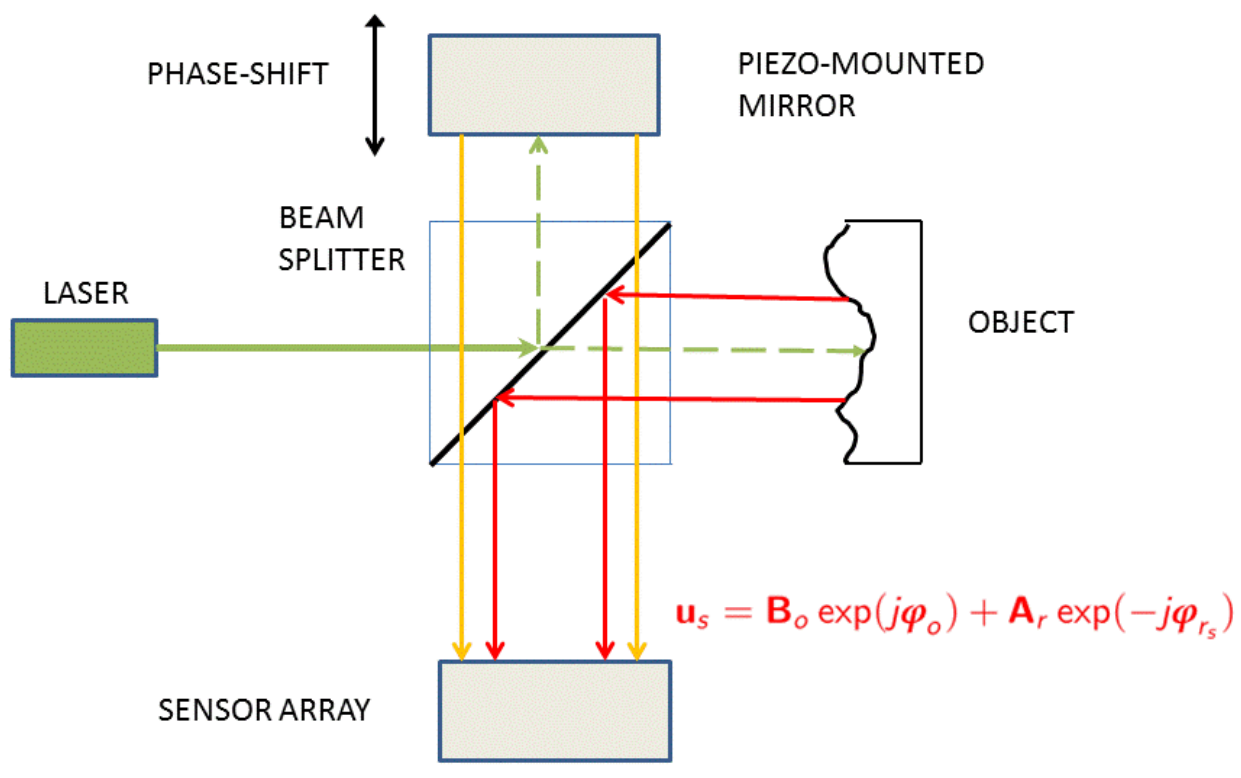

Fig. 1. Phase-shift interferometry: on-axis setup. In this Michelson configuration a single laser beam of coherent light is split into two beams by a beam splitter. The path difference traveled by the reference (mirror) beam and object beam is reflected in the intensity of the aggregated beam $\mathbf{u}_{s}$. This intensity is measured by the sensor array. Shift of the mirror is used in order to introduce different phase shifts between the object and reference beams used in order to reconstruct the object wavefront from the intensity measurements. 


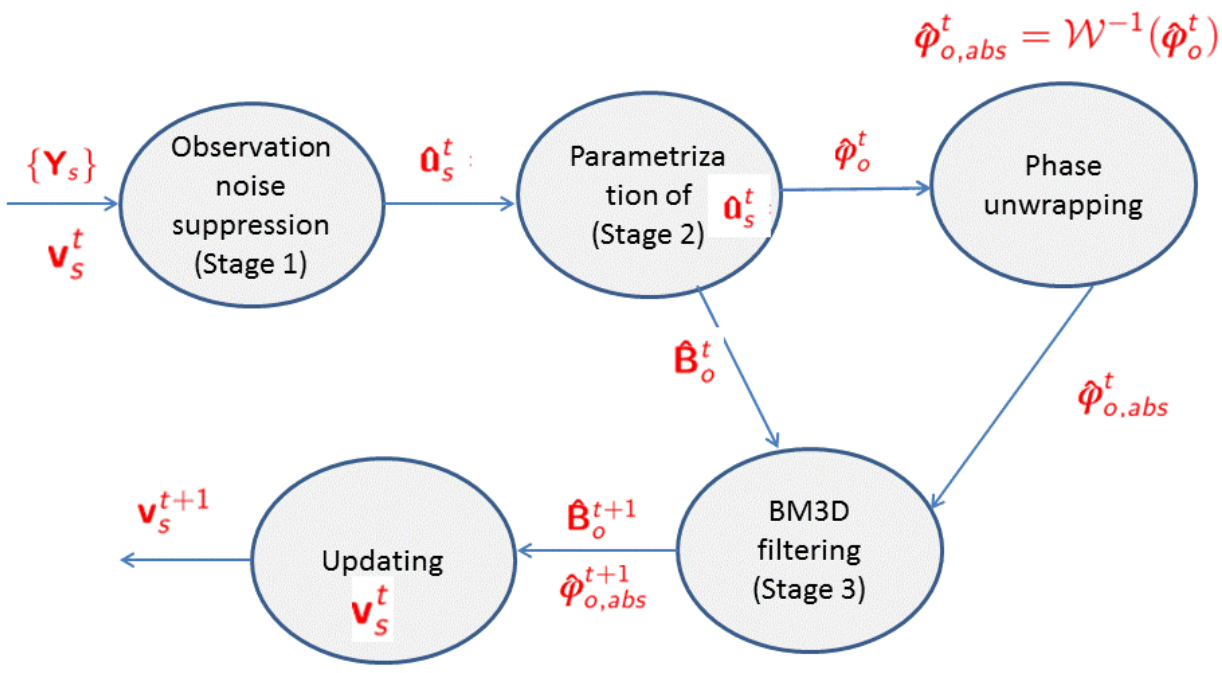

Fig. 2. SPAR algorithm: block-scheme.
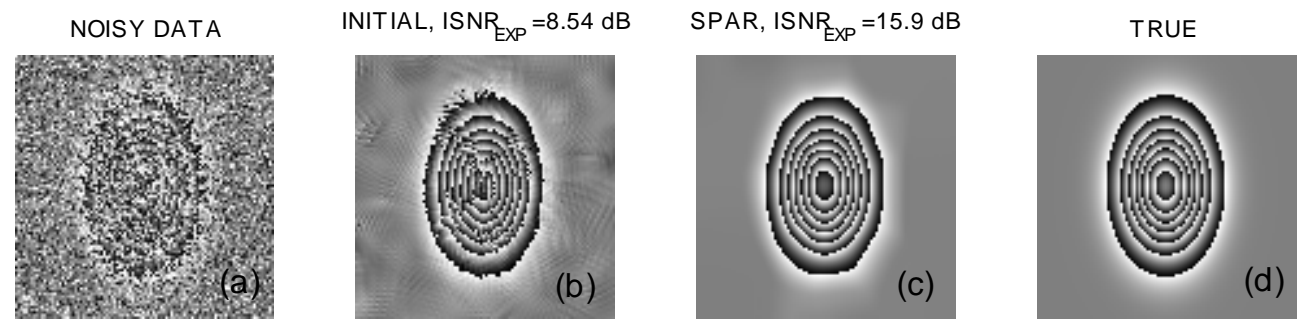

Fig. 3. Wrapped phase for the Gaussian phase object, from left to right: the noisy raw data, the initial $W F T$ estimate, $S P A R$ reconstruction, true image. 

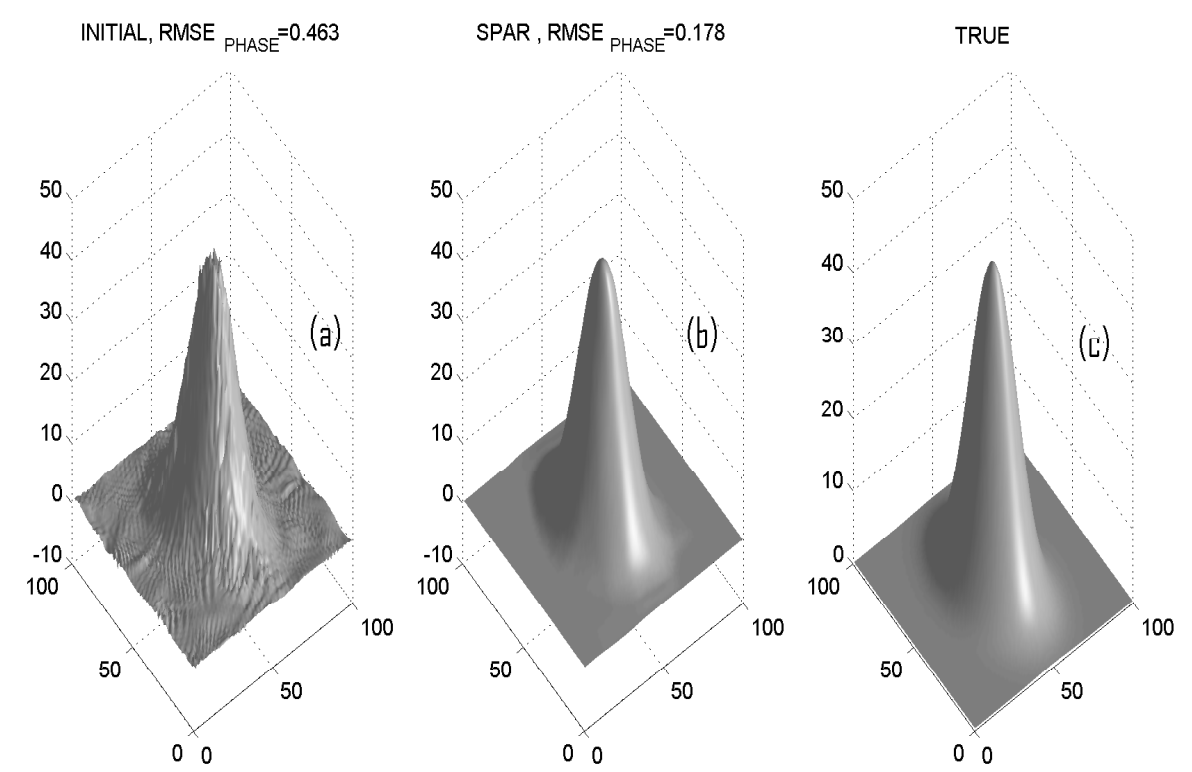

Fig. 4. The absolute (unwrapped) phase for the Gaussian phase object, from left to right: the initial $W F T$ estimate, $S P A R$ reconstruction, true image.
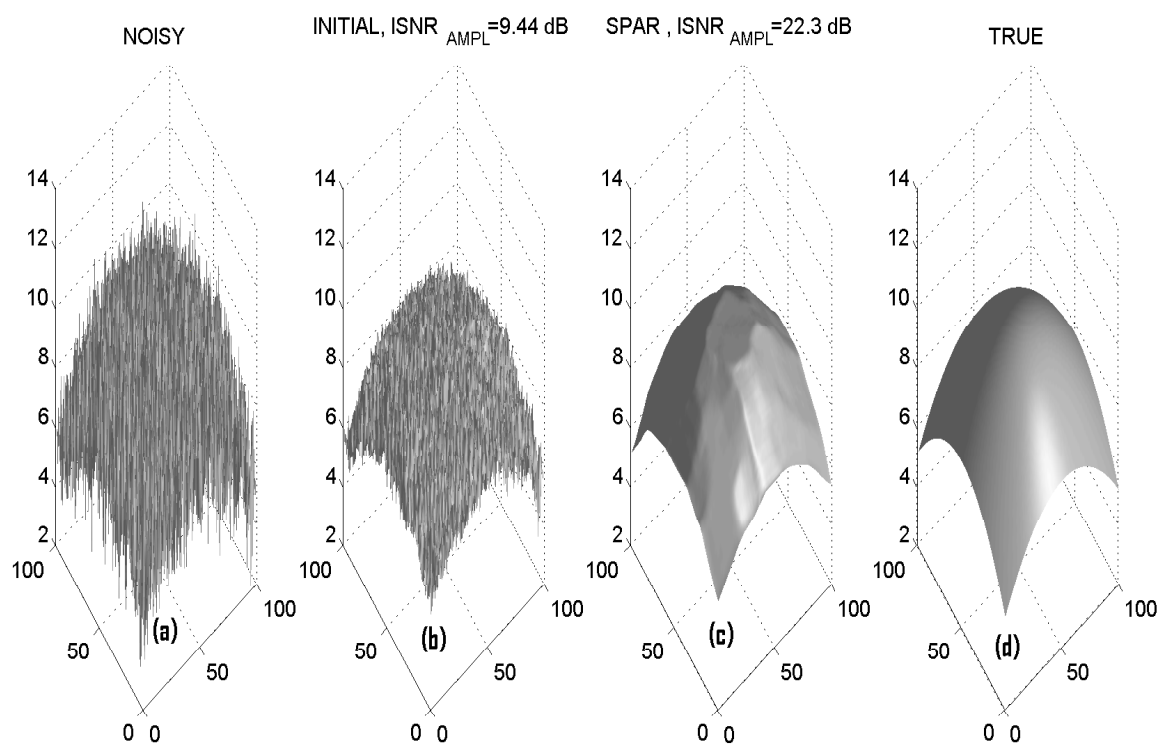

Fig. 5. Surfaces for the quadratic amplitude, from left to right: the noisy raw data, the initial

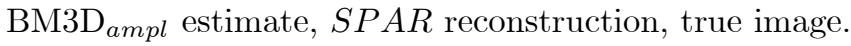



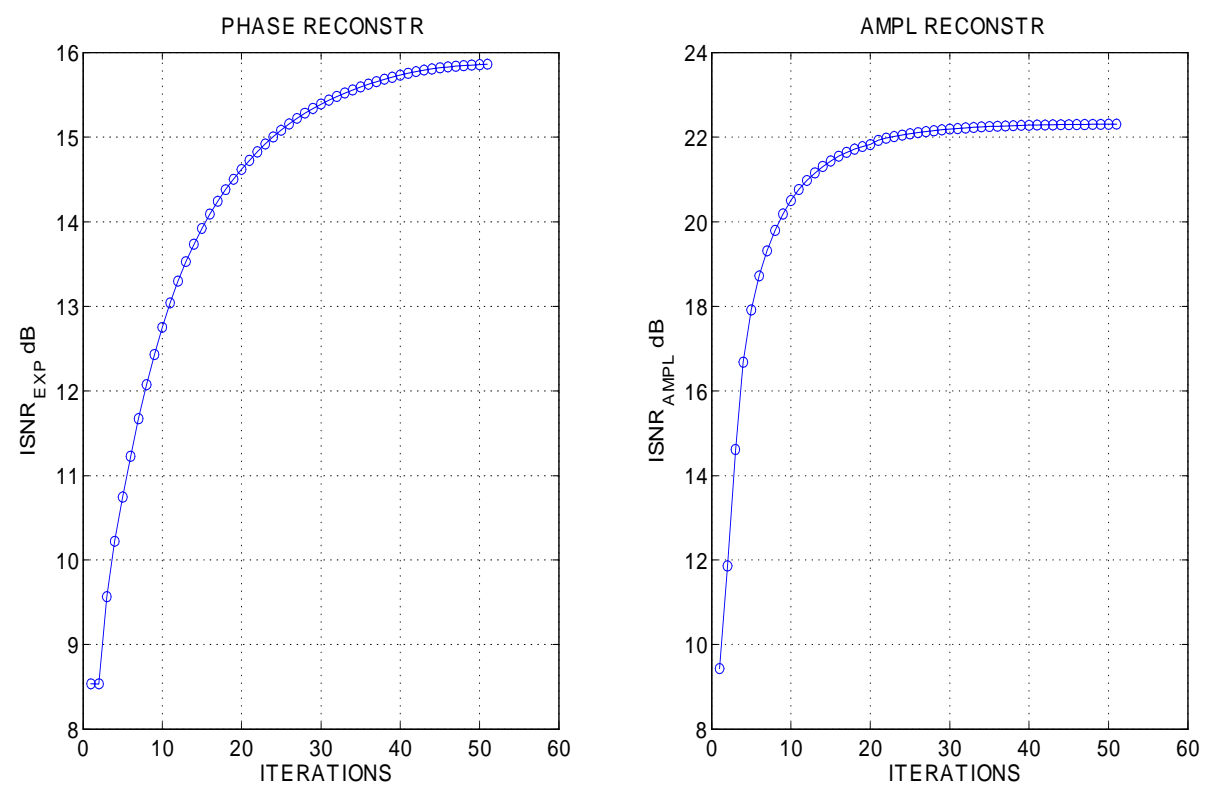

Fig. 6. The accuracy of the wavefront reconstruction (object with the Gaussian phase) versus the number of iterations, from left to right: $I S N R_{\exp }$ for the exponential wavefront $\exp (j \varphi)$, $I S N R_{a m p l}$ for the quadratic amplitude.
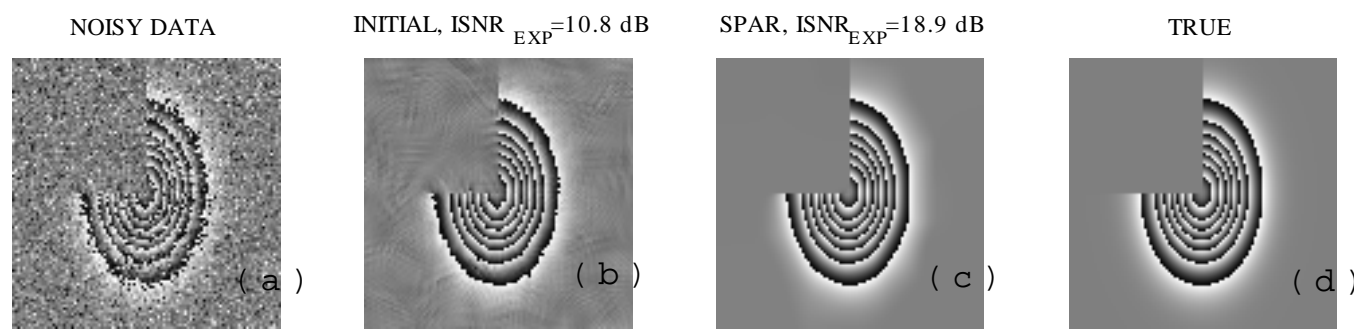

Fig. 7. The wrapped phase for the truncated Gaussian phase object, from left to right: the noisy raw data, the initial $W F T$ estimate, $S P A R$ reconstruction, true image. 

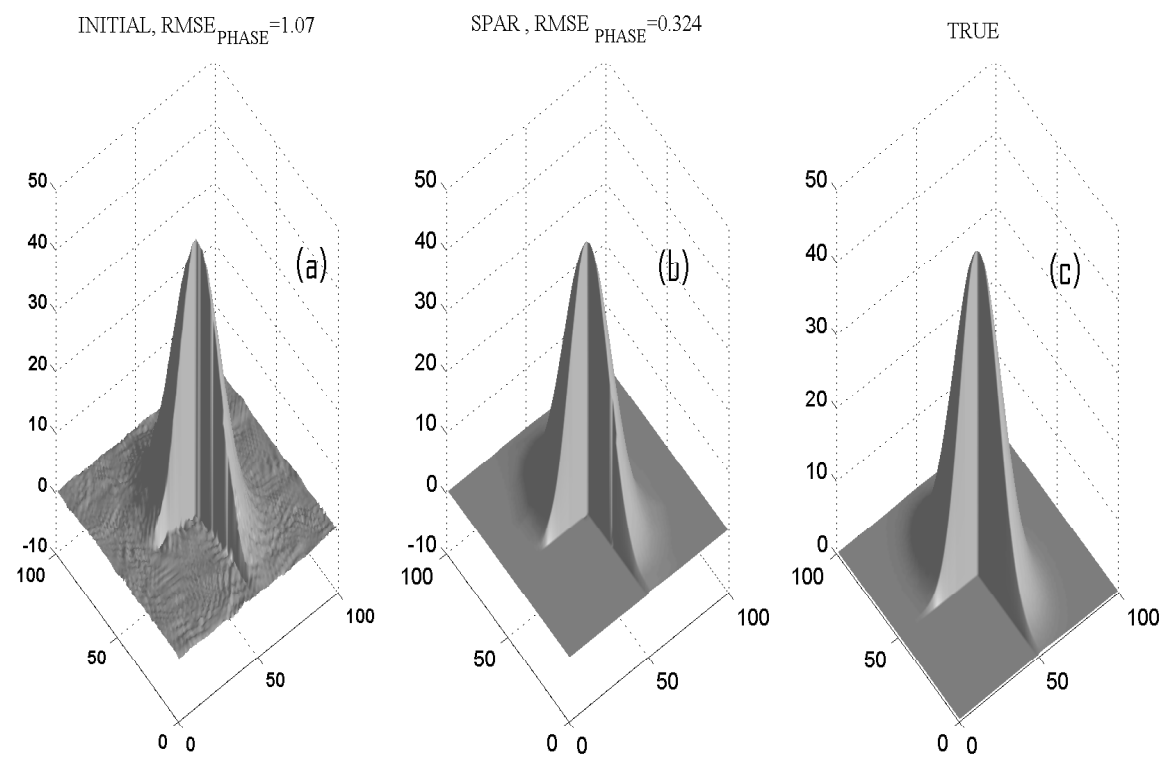

Fig. 8. The absolute (unwrapped) phase for the truncated Gaussian phase object, from left to right: the initial $W F T$ estimate, $S P A R$ reconstruction, true image.
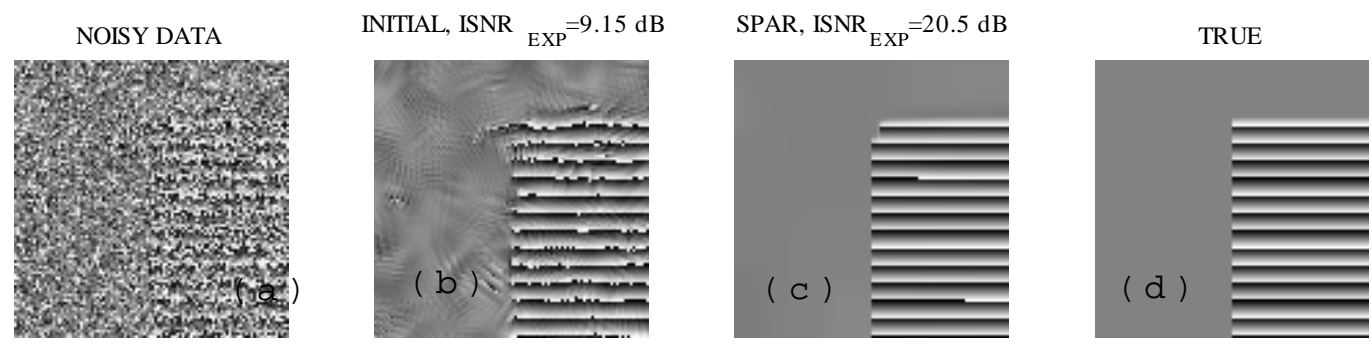

Fig. 9. The wrapped phase for the shear plane phase object, from left to right: the noisy raw data, the initial $W F T$ estimate, $S P A R$ reconstruction, true image. 

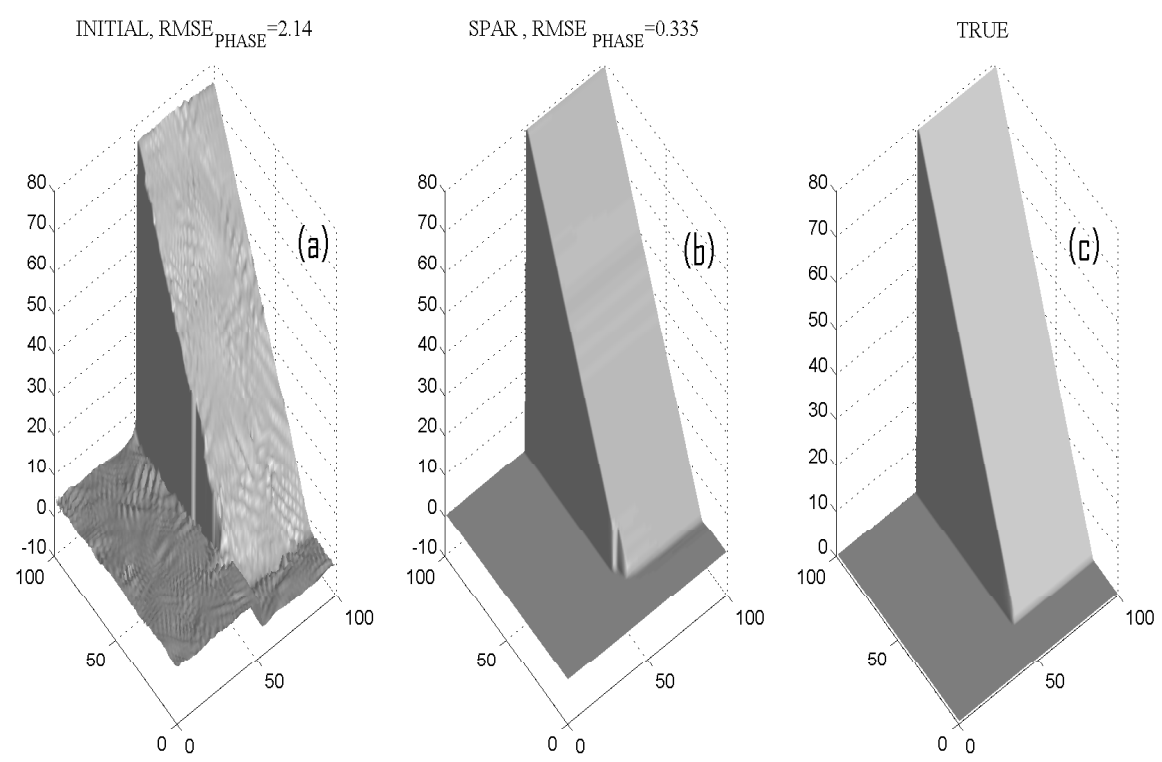

Fig. 10. The absolute (unwrapped) phase for shear plane phase object, from left to right: the initial $W F T$ estimate, $S P A R$ reconstruction, true image. 